\title{
Optical and X-ray activity of the Be/X-ray binary H 1145-619 detected with INTEGRAL
}

\author{
Julia Alfonso-Garzón* \\ Centro de Astrobiología (CSIC-INTA), Camino Bajo del Castillo s/n, Urb. Villafranca del \\ Castillo, E-28692 Villanueva de la Cañada, Spain \\ E-mail: julia@cab.inta-csic.es

\section{Juan Fabregat} \\ Observatorio Astronómico, Universidad de Valencia, Catedrático José Beltrán 2, 46980 \\ Paterna, Spain \\ E-mail: juan.fabregateuv.es
}

\section{Celia Sánchez-Fernández}

European Space Astronomy Centre (ESAC), Camino Bajo del Castillo s/n, Urb. Villafranca del Castillo, E-28692 Villanueva de la Cañada, Spain

E-mail: csanchezesciops.esa.int

\section{Pablo Reig}

IESL, Foundation for Research and Technology-Hellas, 71110, Heraklion, Greece

E-mail: pau@physics.uoc.gr

\section{Jari Kajava}

Tuorla Observatory, Department of Physics and Astronomy, University of Turku, Väisäläntie 20, FIN-21500 Piikkiö, Finland

E-mail: jari.kajava@utu.fi

\section{Lee Townsend}

Department of Astronomy, University of Cape Town, Private Bag X3, Rondebosch, 7701, South Africa

E-mail: townsendeast.uct.ac.za

\section{J. Miguel Mas-Hesse}

Centro de Astrobiología (CSIC-INTA), Camino Bajo del Castillo s/n, Urb. Villafranca del Castillo, E-28692 Villanueva de la Cañada, Spain

E-mail: mmecab.inta-csic.es

\section{Peter Kretschmar}

European Space Astronomy Centre (ESAC), Camino Bajo del Castillo s/n, Urb. Villafranca del Castillo, E-28692 Villanueva de la Cañada, Spain

E-mail: peter.kretschmar@esa.int 
Multiwavelength analysis of Be/X-ray binaries are crucial to understand the mechanism producing their outbursts. H 1145-619 is one of these systems. From 1997 to 2015, this source was believed to be in quiescence, but from the analysis of INTEGRAL/IBIS and JEM-X data, new Xray outbursts in 2003, 2004, and 2005 have been identified. To understand the complex behaviour of the source, optical photometric and spectroscopic observations and measurements from the literature have been compared with the X-ray activity of the source, and a global picture of the optical and X-ray variations during the last 40 years is presented.

11th INTEGRAL Conference Gamma-Ray Astrophysics in Multi-Wavelength Perspective, 10-14 October 2016

Amsterdam, The Netherlands

*Speaker. 


\section{Introduction}

The X-ray pulsar H 1145-619 was discovered in the early 70s with Uhuru (Forman et al., 1978). Pulsations from this source, together with the pulsations from 1E 1145.1-6141 (17' away), were detected in 1977 with Ariel $V$ (White et al., 1978). The two sources were resolved by the Einstein observatory, identifying H 1145-619 as the source of the 292 s pulsations (White et al., 1980; Lamb et al., 1980). A more precise value of the pulse period (292.4 s) was provided by Nagase (1989). The optical counterpart of H 1145-619, Hen 3-715, was discovered in 1978 (Dower et al., 1978) and classified as a 9-mag B1 Ve (Stevens et al., 1997). This system is one of the closest Be/XBs, being located at $3.1 \pm 0.5 \mathrm{kpc}$ from Earth (Stevens et al., 1997). It is a long-period eccentric binary (e $>0.5$, Reig 2011) with an orbital period $\mathrm{P}_{\text {orb }}=186.68 \pm 0.05 \mathrm{~d}$ (Wilson-Hodge, 1999).

Priedhorsky \& Terrell (1983) analysed the X-ray observations of H 1145-619 from 19691976 by the Vela $5 b$ satellite, which revealed an outburst of $600 \mathrm{mCrab}(3-12 \mathrm{keV})$ coming from the source in April 1973. After that, H 1145-619 displayed several X-ray outbursts in 1975-1978, that were observed with Ariel $V$ and Einstein (White et al., 1980; Watson et al., 1981), one of them reaching $600 \mathrm{mCrab}$ in December 1977 (White et al., 1980). X-ray activity was observed again in 1983-1985 with EXOSAT (Cook \& Warwick, 1987). After that, nine consecutive outbursts between 1992 and 1997 were observed by CGRO/BATSE. Two of these outbursts reached peak fluxes of 550 mCrab (in the 20-40 keV band) in March 1994 and October 1996 (Wilson-Hodge, 1999). Three of these nine outbursts were also detected by RXTE/ASM (Corbet \& Remillard, 1996).

In 2015, two X-ray outbursts, coinciding with periastron passages, and with intensities of $\sim 50 \mathrm{mCrab}$ and $\sim 36 \mathrm{mCrab}$ in the $15-50 \mathrm{keV}$ band, were observed by MAXI/GSC and Swift/BAT (Mihara et al., 2015; Nakajima et al., 2015), suggesting renewed X-ray activity of the source.

\section{New X-ray activity discovered with INTEGRAL}

From the late 90's to 2015, the source was believed to be in quiescence in X-rays. However, from the analysis of INTErnational Gamma-Ray Astrophysics Laboratory (INTEGRAL; Winkler et al. 2003) archival data, three outbursts not reported by other observatories have been detected. Using INTEGRAL/JEM-X (Lund et al., 2003) and IBIS (Ubertini et al., 2003) data we found three faint X-ray outbursts in May 2003, June 2004 and June 2005. The fluxes of these outbursts were 33,15 , and $7 \mathrm{mCrab}$ in the $22-40 \mathrm{keV}$ band (measured with INTEGRAL/IBIS), respectively. The intensity of these three outbursts decreased with time, while the duration increased from 2003 to 2005 ( $~ 3$ days in 2003, $\sim 10$ days in 2004, and hard to measure in 2005). The three X-ray outbursts occurred a few days before the corresponding periastron passages, This fact is in agreement with the behaviuor of the outbursts identified by Wilson-Hodge (1999) using BATSE data, which took place within a narrow range of orbital phase (-0.1-0.1). Two of these outbursts have been mis-identified as low luminosity outbursts from the close system 1E 1145.1-6141 (which is at $\sim 17$ arcmin from $\mathrm{H}$ 1145-619) (Ferrigno et al., 2008). We have verified the identification of $\mathrm{H}$ 1145-619 as the active source in these periods generating the IBIS and JEM-X images of the field before and during the outbursts and the light curves of both sources. In Fig. 1, images in outburst (left) and quiescence (right) during the epoch of the 2003 outburst are displayed. From top to bottom 
the energy bands are: 3-10 keV from JEM-X, and 20-35 keV from IBIS/ISGRI. Comparing the images in quiescence with the images in outburst, the brightening of the source in the last ones is evident. The field of view is of $2^{\circ} \times 2^{\circ}$, and H 1145-619 is the source located to the South East appearing only in the outburst images. The source appearing in all the images is 1E 1145.1-6141. In the JEM-X (with an angular resolution is 3 arcmin FWHM) both sources are resolved, and in the images corresponding to the outburst, two point sources are observed. However, in the IBIS/ISGRI images of the outburst epoch, the presence of a new source in the position of $\mathrm{H} 1145-619$ is noticed, but it is blended with 1E 1145.1-6141 due to the poorer resolution (the IBIS angular resolution is 12 arcmin FWHM). The light curves of H 1145-619 with both instruments are also provided in the figure. The observations used to extract the images are marked in grey in the light curves.

\section{Long-term optical and X-ray evolution}

In order to understand the physical mechanisms producing the observed variations at different wavelengths, we have compiled all the available optical and X-ray data from archives and from the literature, and we have combined them with our new observations. A global picture, showing the optical light curves of H 1145-619, the optical spectroscopic results obtained in this work, and the X-ray outbursts from 1982 to 2016 is displayed in Fig. 2. We have included I-Johnson ligth curves from ASAS-2 and ASAS-3 and $V-$ Johnson band light curves from ASAS-3 and INTEGRAL/OMC (Mas-Hesse et al., 2003). The results from the spectroscopic analysis of $\mathrm{H} \alpha$ (in red) and HeI 6678 (in blue) equivalent widths and V/R are displayed in third and fourth respectively. Optical spectroscopic data from the SAAO, SMARTS and SALT telescopes, and from the literature have been included. The X-ray outburts previously reported (detected by BATSE and RXTE in the 80's and 90's), the ones detected with INTEGRAL data in this work, and the last outbursts detected by MAXI and Swift/BAT are marked with dark blue arrows with lengths proportional to the outburst fluxes.

We have observed four different optical patterns of variability: Global increases and decreases of the optical brightness (observed from 1982 to 1994 and since 2009 to 2016), superorbital variations with a period of $\sim 590$ days (observed in 2002-2009), faint and short optical outbursts observed at periastron passages in 1991-1992, and brighter and longer (tens of days) optical outbursts in 1998-1999 and 2002-2005, taking place at different orbital phases.

The optical spectroscopic measurements provide information on the kinematics and the evolution on the circumstellar disc. As can be seen in the EW and V/R light curves, this source displays significant variability in the $\mathrm{H} \alpha$ and $\mathrm{He}$ I $6678 \AA$ line profiles. Maximum values of the $\mathrm{H} \alpha$ emission were measured in the 90's, when an X-ray outburst was observed at every periastron passage. Minimum values of the $\mathrm{H} \alpha$ emission are measured in the period in which the source displays the faintest $V$ - and $I$-band brightnesses (1998-2009) and almost no X-ray outbursts are observed (only the faint X-ray outbursts detected with INTEGRAL in this work).

\section{Discussion}

From the historical multiwavelength analysis of H 1145-619, we can infer some physical properties of the circumstellar disc and its repercussion on the X-ray activity. 

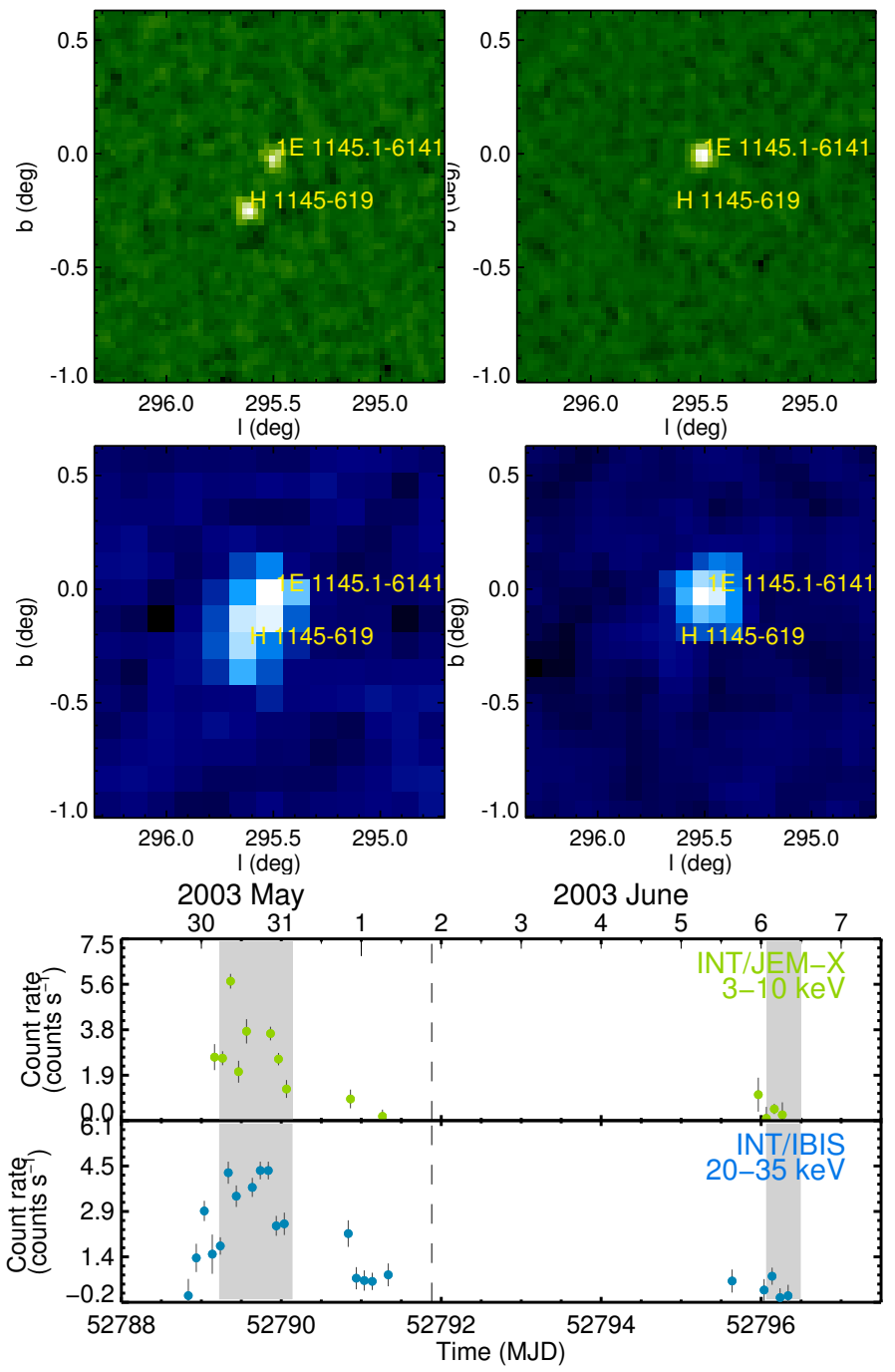

Figure 1: In green, the JEM-X images in the 3-10 keV energy band and in blue the IBIS/ISGRI images in the 20-35 keV energy range of $\mathrm{H} \mathrm{1145-619} \mathrm{in} \mathrm{outburst} \mathrm{(left)} \mathrm{and} \mathrm{quiescence} \mathrm{(right)} \mathrm{in} \mathrm{the} \mathrm{epoch} \mathrm{of} \mathrm{the} 2003$ outburst. The field of view is of $2^{\mathrm{O}} \times 2^{\mathrm{O}}$, and $\mathrm{H}$ 1145-619 is the source located more to the South and to the East appearing only in the images from the outburst, and the other one appearing in all the images is 1E 1145.1-6141. At the bottom, the corresponding light curves of H 1145-619 are plotted. The data used to extract the images are marked with a grey shadow.

In the 80's and 90's, the optical continuum varies smoothly between $V \sim 9.4$ and $V \sim 8.8$. This variability can be explained by the evolution of the circumstellar disc. In 1991-1992, two faint optical outbursts are observed at periastron passages, and coinciding with X-ray outbursts, so they should be related with the interaction of the neutron star with the disc.

From 1991 to 1997 H 1145-619 showed an X-ray outburst at every periastron passage, and after that it went to quiescence. From spectroscopic measurements from 1993 to 1999, we see that the EW of the emission lines decreased from values around $-35 \AA$ to values around $-7 \AA$ in this period. This drop of the $\mathrm{H} \alpha$ and $\mathrm{HeI}$ emission, which is in agreement with decay observed in the 


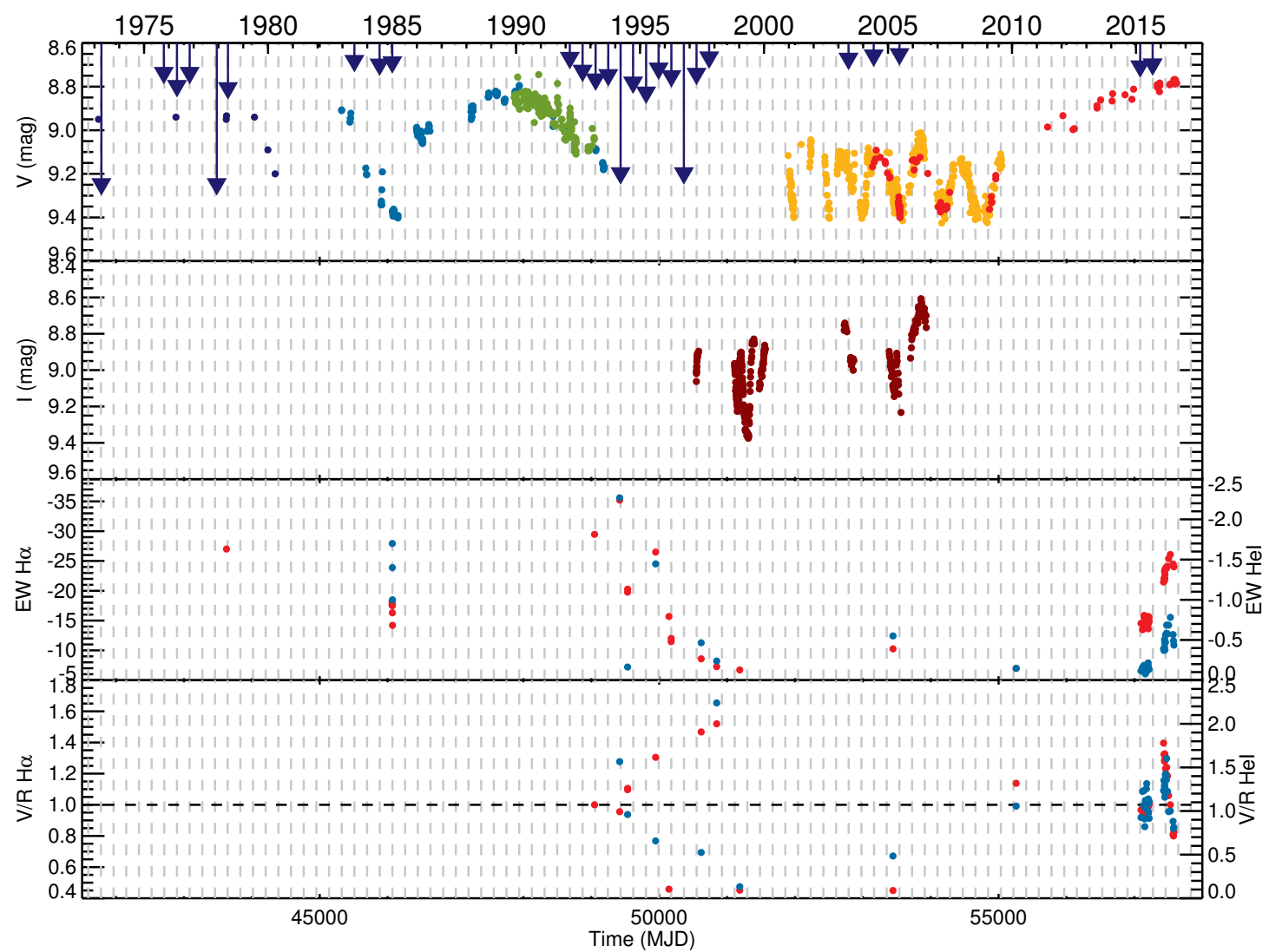

Figure 2: Optical light curves of H 1145-619 from 1973 to 2015. The periastron passages were calculated using the ephemeris $\mathrm{P}_{\text {orb }}=186.68 \mathrm{~d} ; \mathrm{T}_{0}=$ MJD 48871.6 (Wilson-Hodge, 1999) and are marked with grey dashed lines (they are marked in all the light curves in the paper). The X-ray outbursts are marked with dark blue arrows with lengths proportional to the outburst fluxes. First panel: $V$ - Johnson optical light curves. Dark blue points come from the literature (Jones et al., 1974; Hammerschlag-Hensberge et al., 1980; Pakull et al., 1980), light blue points are from the ESO's Long-Term Photometric Variable Project (Sterken et al., 1995), green points are from Hipparcos (Perryman et al., 1997), orange points come from ASAS-3 and the red points from INTEGRAL/OMC data. Second panel: $I$-band photometric points from ASAS-2. Third panel: EW for $\mathrm{H} \alpha$ (red points) and He I $6678 \AA$ (blue points). We have included 1978 and 1985 measurements by Jones et al. (1974) and Cook \& Warwick (1987) respectively, we have re-analysed the observations from 1993 to 1996 published in Stevens et al. (1997), and we present in this work new data and results from 1997 to 2016. Fourth panel: V/R for the same spectra (see text).

$V$-band optical brightness, is usually interpreted as dissipation of the circumstellar disc.

From 2002 to 2009, the optical brightness remains substantially lower than in the other periods, with a mean value of $V \sim 9.3$ mag. Appart from the observed fainter optical emission, the source displayed superorbital modulations. Superorbital variations in Be/X-ray binaries can be interpreted as the propagation of density waves. Overimposed to them, three optical outbursts previous to the high-energy from 2003-2005 reported in this work. They could be produced by mass ejections. 
From 2009 to 2016, there has been a substantial increase of the optical flux ( $\sim 0.5 \mathrm{mag}$ ), probably caused by a new enhancement of the disc (Okazaki \& Negueruela, 2001; Haigh et al., 2004).

From 2015 to mid 2016, the optical magnitude has remained stable and close to $V \sim 8.8$ mag, similar to the brightness measured by Stevens et al. (1997) in the 90's, so the disc should have similar physical properties. We should expect then, a renewed X-ray activity of the source. Indeed, during the two periastron passages in 2015, two normal X-ray outbursts were observed (Mihara et al., 2015; Nakajima et al., 2015). We have INTEGRAL and Swift approved ToO proposals in case this system undergoes a new outburst in 2017 and we will continue the optical monitoring to obtain information on the evolution of the disc.

A detailed analysis of these observations and a deeper interpretation of the optical and X-ray correlations observed in this system are presented in Alfonso-Garzón et al. (2017).

\section{References}

Alfonso-Garzón, J., Fabregat, J., Reig, P., Kajava, J. J. E., Sánchez-Fernández, C., Townsend, L. J., Mas-Hesse, J. M., Crawford, S. M., Kretschmar, P., Coe, M. J., submitted, A\&A

Cook, M. C. \& Warwick, R. S. 1987, Monthly Notices of the Royal Astronomical Society, 227, 661

Corbet, R. \& Remillard, R. 1996, International Astronomical Union Circular, 6486, 1

Dower, R. G., Bradt, H. V., Doxsey, R. E., et al. 1978, Nature, 273, 364

Ferrigno, C., Segreto, A., Mineo, T., Santangelo, A., \& Staubert, R. 2008, A\&A, 479, 533

Forman, W., Jones, C., Cominsky, L., et al. 1978, The Astrophysical Journal Supplement Series, 38,357

Haigh, N. J., Coe, M. J., \& Fabregat, J. 2004, Monthly Notices of the Royal Astronomical Society, 350,1457

Hammerschlag-Hensberge, G., van den Heuvel, E. P. J., Lamers, H. J. G. L. M., et al. 1980, Astronomy and Astrophysics, 85, 119

Jones, C. A., Chetin, T., \& Liller, W. 1974, The Astrophysical Journal Letters, 190, L1

Lamb, R. C., Markert, T. H., Hartman, R. C., Thompson, D. J., \& Bignami, G. F. 1980, ApJ, 239, 651

Lund, N., Budtz-J?rgensen, C., Westergaard, N. J., et al. 2003, Astronomy and Astrophysics, 411, L231

Mas-Hesse, J. M., Giménez, A., Culhane, J. L., et al. 2003, Astronomy and Astrophysics, 411, L261

Mihara, T., Negoro, H., Krimm, H. A., et al. 2015, ATel, 7215, 1 
Nagase, F. 1989, PASJ, 41, 1

Nakajima, M., Mihara, T., Ueno, S., et al. 2015, ATel, 7015, 1

Okazaki, A. T. \& Negueruela, I. 2001, A\&A, 377, 161

Pakull, M., Lub, J., \& Reipurth, B. 1980, International Astronomical Union Circular, 3476, 1

Perryman, M. A. C., Lindegren, L., Kovalevsky, J., et al. 1997, A\&A, 323, L49

Priedhorsky, W. C. \& Terrell, J. 1983, ApJ, 273, 709

Reig, P. 2011, Astrophysics and Space Science, 332, 1

Sterken, C., Manfroid, J., Beele, D., et al. 1995, Astronomy and Astrophysics Supplement Series, 113,31

Stevens, J. B., Reig, P., Coe, M. J., et al. 1997, MNRAS, 288, 988

Ubertini, P., Lebrun, F., Di Cocco, G., et al. 2003, Astronomy and Astrophysics, 411, L131

Watson, M. G., Warwick, R. S., \& Ricketts, M. J. 1981, MNRAS, 195, 197

White, N. E., Becker, R. H., Pravdo, S. H., et al. 1980, ApJ, 239, 655

White, N. E., Parkes, G. E., Sanford, P. W., Mason, K. O., \& Murdin, P. G. 1978, Nature, 274, 664

Wilson-Hodge, C. A. 1999, PhD thesis, University of Alabama in Huntsville

Winkler, C., Courvoisier, T. J., Di Cocco, G., et al. 2003, Astronomy and Astrophysics, 411, L1 